\title{
Micropolar flow in a porous channel with high mass transfer
}

\author{
N. A. Kelson* A. Desseaux ${ }^{\dagger} \quad$ T. W. Farrell
}

(Received 1 October 2001; revised 25 October 2002)

\begin{abstract}
Two dimensional flow of a micropolar fluid in a porous channel is investigated. The flow is driven by suction or injection at the channel walls, and the micropolar model due to Eringen is used to describe the working fluid. An extension of Berman's similarity transform is used to reduce the governing equations to a set of non-linear coupled ordinary differential equations. The latter are solved for large mass transfer via a perturbation analysis where the inverse of the cross-flow Reynolds number is used as the perturbing parameter. Complementary numerical solutions for strong injection are also obtained using a quasilinearisation scheme, and good agreement is observed between the solutions obtained from the perturbation analysis and the computations.
\end{abstract}

*HPC and Research Support, QUT, AUSTRALIA.

mailto:n.kelson@qut.com

$\dagger$ Institut Universitaire de Technologie, Valenciennes, FrancE.

${ }^{\ddagger}$ Centre in Statistical Science and Industrial Mathematics, QUT, Australia.

${ }^{0}$ See http://anziamj.austms.org.au/V44/CTAC2001/Kels for this article, (C) Austral. Mathematical Soc. 2003. Published 1 April 2003. ISSN 1446-8735 


\section{Contents}

1 Introduction

C481

2 Defining equations

C482

3 Perturbation analysis

C485

4 Numerical method

C487

5 Results and discussion

C489

6 Conclusion

C493

References

C493

\section{Nomenclature}

\begin{tabular}{cl}
\hline$j$ & micro-inertia density $\left(\mathrm{m}^{2}\right)$ \\
$q$ & mass transfer parameter $\left(\mathrm{m} \mathrm{s}^{-1}\right)$ \\
$s$ & microrotation boundary condition parameter \\
$u, v$ & cartesian velocity components $\left(\mathrm{m} \mathrm{s}^{-1}\right)$ \\
$x, y$ & cartesian coordinate parallel and normal to channel $(\mathrm{m})$ \\
$F, G$ & dimensionless stream function and microrotation \\
$N$ & microrotation/angular velocity $\left(\mathrm{s}^{-1}\right)$ \\
$N_{1,2,3}$ & dimensionless parameters \\
$\eta$ & dimensionless normal distance \\
$\mu, \kappa$ & dynamic viscosity and coupling coefficient $\left(\mathrm{kg} \mathrm{m}^{-1} \mathrm{~s}^{-1}\right)$ \\
$\nu$ & kinematic viscosity $\left(\mathrm{m}^{2} \mathrm{~s}^{-1}\right)$ \\
$\nu_{s}$ & microrotation/spin-gradient viscosity $\left(\mathrm{m} \mathrm{kg} \mathrm{s}^{-1}\right)$ \\
$\rho$ & fluid density $\left(\mathrm{kg} \mathrm{m}^{-3}\right)$ \\
\hline
\end{tabular}




\section{Introduction}

The theory of micropolar fluids was originally formulated by Eringen [4]. In essence, the theory introduces new material parameters, an additional independent vector field - the microrotation - and new constitutive equations which must be solved simultaneously with the usual equations for Newtonian flow. The desire to model the non-Newtonian flow of fluids containing rotating microconstituents provided initial motivation for the development of the theory, but subsequent studies have successfully applied the model to a wide range of applications including blood flow, lubricants, porous media, turbulent shear flows, and flow in capillaries and microchannels. For a resume of the theory and more recent literature see the book by Lukaszewicz [10].

We analyse self-similar boundary layer flow of a micropolar fluid in a porous channel, where the flow is driven by uniform mass transfer through the channel walls. The corresponding Newtonian fluid model was first studied by Berman [1], who described an exact solution of the Navier-Stokes equations by assuming a self-similar solution and reducing the governing partial differential equations to a nonlinear ordinary differential equation of fourth order. The solution is of potential value in understanding more realistic flows in channels and pipes, and study of Berman's exact solution and generalisations of it have attracted numerous studies subsequently, for example Yuan [15], Robinson [14], Zaturska et al. [16], Desseaux [2].

With a view to understanding how the micropolar theory may be used to model realistic non-Newtonian flow applications, the authors have considered micropolar extensions to this and other problems, see for example, Kelson \& Desseaux [7], Kelson \& Farrell [8], Desseaux \& Kelson [3]. In the absence of extensive experimental data for micropolar fluids, the aim of this paper is primarily the- 
oretical: we wish to determine how the material constants of the micropolar fluid influence the flow for large mass transfer through the channel walls. To this end, a perturbation scheme based on the inverse of the cross-flow Reynolds number and numerical quasilinearisation scheme are employed here.

This paper is organised as follows. In section 2 the governing equations and similarity transformations are presented, and appropriate values for the physical parameters are discussed. In section 3, a perturbation analysis is used to derive closed form solutions to first order, and the numerical methods used here are briefly described in section 4. The analytic and numerical results are discussed in section 5, and finally, the conclusions of this study are summarised in section 6 .

\section{Defining equations}

We consider steady, incompressible, laminar flow of a micropolar fluid along a two-dimensional channel with porous walls through which fluid is uniformly injected or removed with speed $q$. Using cartesian coordinates, the channel walls are parallel to the $x$-axis and located at $y= \pm h$, where $2 h$ is the channel width.

The relevant equations governing the flow are (see, for example, 
Ramachandran et al. [13]):

$$
\begin{aligned}
\frac{\partial u}{\partial x}+\frac{\partial v}{\partial y} & =0 \\
u \frac{\partial u}{\partial x}+v \frac{\partial u}{\partial y} & =-\frac{1}{\rho} \frac{\partial p}{\partial x}+\left(\nu+\frac{\kappa}{\rho}\right)\left(\frac{\partial^{2} u}{\partial x^{2}}+\frac{\partial^{2} u}{\partial y^{2}}\right)+\frac{\kappa}{\rho} \frac{\partial N}{\partial y} \\
u \frac{\partial v}{\partial x}+v \frac{\partial v}{\partial y} & =-\frac{1}{\rho} \frac{\partial p}{\partial y}+\left(\nu+\frac{\kappa}{\rho}\right)\left(\frac{\partial^{2} v}{\partial x^{2}}+\frac{\partial^{2} v}{\partial y^{2}}\right)-\frac{\kappa}{\rho} \frac{\partial N}{\partial x} \\
u \frac{\partial N}{\partial x}+v \frac{\partial N}{\partial y} & =-\frac{\kappa}{\rho j}\left(2 N+\frac{\partial u}{\partial y}-\frac{\partial v}{\partial x}\right)+\frac{\nu_{s}}{\rho j}\left(\frac{\partial^{2} N}{\partial x^{2}}+\frac{\partial^{2} N}{\partial y^{2}}\right) .
\end{aligned}
$$

Compared with Newtonian fluids, the governing equations include the microrotation or angular velocity $N$ whose direction of rotation is in the $x y$-plane, and the material parameters $j, \kappa$ and $\nu_{s}$. For consistency with other micropolar studies, all material parameters are taken as independent and constant. When these constants are zero, the governing equations reduce to those given by Berman [1].

The appropriate physical boundary conditions are

$$
u(x, \pm h)=0, \quad v(x, \pm h)= \pm q, \quad N(x, \pm h)=-\left.s \frac{\partial u}{\partial y}\right|_{(x, \pm h)}
$$

and assuming that the flow is symmetric about $y=0$,

$$
\frac{\partial u}{\partial y}(x, 0)=v(x, 0)=0,
$$

where $q>0$ corresponds to suction, $q<0$ to injection, and $s$ is a boundary parameter that is used to model the extent to which microelements are free to rotate in the vicinity of the channel walls. For example, the value $s=0$ corresponds to the case where microelements close to a wall are unable to rotate, whereas the value $s=1 / 2$ corresponds to the case where the microrotation is equal to the fluid vorticity at the boundary (see Lukaszewicz [10]). 
To simplify the governing equations, we generalise Berman's similarity solution [1] to include micropolar effects by assuming a stream function and microrotation of the form

$$
\Psi=-q x F(\eta), \quad N=\frac{q x}{h^{2}} G(\eta),
$$

where

$$
\eta=y / h, \quad u=\frac{\partial \Psi}{\partial y}=-\frac{q x}{h} F^{\prime}(\eta), \quad v=-\frac{\partial \Psi}{\partial x}=q F(\eta) .
$$

In addition, we introduce the dimensionless micropolar parameters and non-zero cross-flow Reynolds number

$$
N_{1}=\frac{\kappa}{\rho \nu}, \quad N_{2}=\frac{\nu_{s}}{\rho \nu h^{2}}, \quad N_{3}=\frac{j}{h^{2}}, \quad \text { and } \quad \operatorname{Re}=\frac{q h}{\nu}
$$

where $\operatorname{Re}>0$ corresponds to suction, and $\operatorname{Re}<0$ to injection.

Substituting (6) and (7) into (1-4) reduces the governing equations to $\partial^{2} p / \partial x \partial \eta=0$,

$$
\operatorname{Re}\left[F F^{\prime \prime \prime}-F^{\prime} F^{\prime \prime}\right]=\left(1+N_{1}\right) F^{I V}-N_{1} G^{\prime \prime}
$$

or, after integrating once for some constant $K$,

$$
\operatorname{Re}\left[F F^{\prime \prime}-\left(F^{\prime}\right)^{2}\right]=\left(1+N_{1}\right) F^{\prime \prime \prime}-N_{1} G^{\prime}+K,
$$

and

$$
N_{3} \operatorname{Re}\left(F G^{\prime}-F^{\prime} G\right)=N_{1}\left(F^{\prime \prime}-2 G\right)+N_{2} G^{\prime \prime} .
$$

Using $\epsilon=1 / \operatorname{Re},(8 b)$ and (9) are written

$$
\begin{aligned}
F F^{\prime \prime}-\left(F^{\prime}\right)^{2} & =\epsilon\left(1+N_{1}\right) F^{\prime \prime \prime}-\epsilon N_{1} G^{\prime}+C, \\
N_{3}\left(F G^{\prime}-F^{\prime} G\right) & =\epsilon N_{1}\left(F^{\prime \prime}-2 G\right)+\epsilon N_{2} G^{\prime \prime},
\end{aligned}
$$


where $C=K /$ Re is to be determined as a function of the boundary conditions. The latter are

$$
F( \pm 1)=1, \quad F^{\prime}( \pm 1)=0, \quad G( \pm 1)=s F^{\prime \prime}( \pm 1),
$$

or assuming symmetric flow in the channel

$$
F(0)=F^{\prime \prime}(0)=F^{\prime}(1)=0, \quad F(1)=1, \quad G(1)=s F^{\prime \prime}(1) .
$$

For a given cross-flow Reynolds number Re, the problem as defined contains four additional micropolar parameters $s, N_{1,2,3}$. In general, the parameters may depend on the concentration and shape of the microelements, and a determination of the parameter values is a difficult matter [5]. The experimental work of Migun and colleagues (see, for example, Migun [12], Kolpashchikov et al. [9], Migoun \& Prokhorenko [11]) suggests that $N_{1}=\mathcal{O}(1)$ for water in capillary tubes, whereas $N_{2}$ and $N_{3}$ must be non-negative for micropolar fluids [4]. For comparison with existing studies, in this work we set $s=0, N_{1,2}=1, N_{3}=10^{-1}$ and investigate solution behaviour as Re is varied.

\section{$3 \quad$ Perturbation analysis}

The solution of (10) and (11) is sought by substituting series expansions for $F, G$ and $C$ near $\epsilon=0$ of the form

$$
\begin{aligned}
& F=f_{0}+\epsilon f_{1}+\epsilon^{2} f_{2}+\cdots, \\
& G=g_{0}+\epsilon g_{1}+\epsilon^{2} g_{2}+\cdots, \\
& C=c_{0}+\epsilon c_{1}+\epsilon^{2} c_{2}+\cdots,
\end{aligned}
$$

and collecting terms in equal powers of $\epsilon$ to obtain a heirachy of ordinary differential equations for the functions $f_{n}$ and $g_{n}$. The 
first two equations for $f_{n}$ are

$$
\begin{aligned}
f_{0} f_{0}^{\prime \prime}-\left(f_{0}^{\prime}\right)^{2} & =c_{0}, \\
f_{0} f_{1}^{\prime \prime}-2 f_{0}^{\prime} f_{1}^{\prime}+f_{0}^{\prime \prime} f_{1} & =c_{1}+\left(1+N_{1}\right) f_{0}^{\prime \prime \prime}-N_{1} g_{0}^{\prime},
\end{aligned}
$$

and the corresponding equations for $g_{n}$ are

$$
\begin{aligned}
f_{0} g_{0}^{\prime}-f_{0}^{\prime} g_{0} & =0 \\
N_{3}\left(f_{0} g_{1}^{\prime}-f_{0}^{\prime} g_{1}\right) & =N_{3}\left(f_{1}^{\prime} g_{0}-f_{1} g_{0}^{\prime}\right)+N_{1}\left(f_{0}^{\prime \prime}-2 g_{0}\right)+N_{2} g_{0}^{\prime \prime} .
\end{aligned}
$$

Assuming that the various terms in (10) and (11) are of similar size, we anticipate that equations (14) and (15) apply provided that $N_{1}<|\operatorname{Re}|, N_{3} \neq 0$ and $N_{3}>|\epsilon| N_{1,2}$.

The boundary conditions for equations (14) and (15) are

$$
\begin{array}{ll}
f_{0}(0)=f_{0}^{\prime \prime}(0)=f_{0}^{\prime}(1)=0, & f_{0}(1)=1, \quad g_{0}(1)=s f_{0}^{\prime \prime}(1) \\
f_{n}(0)=f_{n}^{\prime \prime}(0)=f_{n}^{\prime}(1)=0, & f_{n}(1)=0, \quad g_{n}(1)=s f_{n}^{\prime \prime}(1)
\end{array}
$$

for $n>0$.

The leading order solutions $f_{0}$ and $g_{0}$ to equations (14a) and (15a) that satisfy all the required boundary conditions (16a) are

$$
f_{0}(\eta)=\sin \left(\frac{\pi \eta}{2}\right), \quad g_{0}(\eta)=-\frac{1}{4} s \pi^{2} \sin \left(\frac{\pi \eta}{2}\right), \quad c_{0}=-\frac{\pi^{2}}{4} .
$$

Note that the ordinary differential equation (14b) for the first order stream function is linear and second order with variable coefficients. Using the solutions for $f_{0}$ and $g_{0}$ given in (17) and equating the RHS of (14b) to zero, the general solution to the resulting homogeneous equation is of the form

$$
c_{12} \cos \left(\frac{\pi \eta}{2}\right)+c_{13}\left[\frac{2}{\pi} \sin \left(\frac{\pi \eta}{2}\right)-\eta \cos \left(\frac{\pi \eta}{2}\right)\right],
$$


where $c_{12,13}$ are constants. Using techniques such as reduction of order or variation of parameters $[17$, e.g.], a solution of $(14 \mathrm{~b})$ is

$$
\begin{aligned}
f_{1}= & -\frac{2}{\pi^{2}} c_{1} \sin \left(\frac{\pi \eta}{2}\right)+\left[c_{12}+\frac{\tilde{A}}{2} \int_{1}^{\eta} \frac{r}{\sin (\pi r / 2)} d r\right] \cos \left(\frac{\pi \eta}{2}\right) \\
& +\left[c_{13}+\frac{\tilde{A}}{\pi} \log \left|\tan \left(\frac{\pi \eta}{4}\right)\right|\right]\left[\frac{2}{\pi} \sin \left(\frac{\pi \eta}{2}\right)-\eta \cos \left(\frac{\pi \eta}{2}\right)\right],
\end{aligned}
$$

where $\tilde{A}=\frac{\pi^{3}}{8}\left[1+N_{1}(1-s)\right]$.

In general, a solution for $f_{1}$ with four arbitrary constants is required. In the present case, the condition $f_{1}^{\prime \prime}(0)=0$ in $(16 \mathrm{~b})$ is redundant, and the values of the constants for a symmetric solution satisfying all the boundary conditions are

$$
\begin{aligned}
& c_{1}=-\frac{2}{\pi} \tilde{A}\left(1+\frac{\pi^{2}}{4} I_{0}\right), \quad c_{12}=-\frac{\tilde{A}}{2} I_{0}, \quad c_{13}=\frac{c_{1}}{\pi} \\
& \text { and } I_{0}=\int_{1}^{0} \frac{r}{\sin (\pi r / 2)} d r \approx-0.742454 .
\end{aligned}
$$

The solutions described above are exact for the limiting case $N_{1}=$ 0 , and the first order solution $F \approx f_{0}+\epsilon f_{1}$ is expected to provide a good approximation to the exact solution for large mass transfer.

\section{Numerical method}

For the computational study we sought the solution of the coupled two-point non-linear boundary value problem (8a), (9) and (12a). 
Our previous experience [6, e.g.] suggests that a readily adaptable and robust approach to the numerical solution of micropolar flow problems of this type is provided by an iterative scheme based on quasilinearisation.

In this approach we let

$$
\left\{W_{k}: k=1, \ldots, 6\right\}=\left\{G, G^{\prime}, F, F^{\prime}, F^{\prime \prime}, F^{\prime \prime \prime}\right\}
$$

be an approximate current solution and $\left\{Z_{k}: k=1, \ldots, 6\right\}$ be an improved solution for $F$ and $G$ in equations (8a) and (9). The latter are rewritten as a coupled first order system of the form $Z_{k}^{\prime}=L_{k}\left(Z_{1}, Z_{2}, \ldots, Z_{6}\right)$ and linearised by forming a Taylor series expansion around the current estimate $W_{k}$ and neglecting second and higher derivative terms, that is,

$$
Z_{k}^{\prime}=L_{k}\left(W_{1}, W_{2}, \ldots, W_{6}\right)+\left.\sum_{j=1}^{6}\left(Z_{j}-W_{j}\right) \frac{\partial L_{k}}{\partial Z_{j}}\right|_{Z_{j}=W_{j}}
$$

For example, the linearisation of (9) is obtained by setting $N_{2} L_{2}=$ $N_{3} \operatorname{Re}\left(Z_{3} Z_{2}-Z_{4} Z_{1}\right)+N_{1}\left(2 Z_{1}-Z_{5}\right)$ and using equation (21) to yield

$$
\begin{aligned}
N_{2} Z_{2}^{\prime} & =N_{3} \operatorname{Re}\left(Z_{2} W_{3}+Z_{3} W_{2}-Z_{1} W_{4}-Z_{4} W_{1}\right) \\
& +N_{1}\left(2 Z_{1}-Z_{5}\right)+N_{3} \operatorname{Re}\left(W_{4} W_{1}-W_{3} W_{2}\right) .
\end{aligned}
$$

To solve for the $Z_{k}$ at each iteration, the solutions to four separate initial value problems (IVP), denoted $\left\{Z_{k}^{i}: i=0, \ldots, 3\right\}$, with initial conditions given in Table 1 are first obtained using a Runge-Kutta scheme. The solution $Z_{k}$ is then formed via a linear combination of the $Z_{k}^{i}$ such that the required conditions at the second boundary are satisfied.

This solution is then compared with the solution at the previous step, and a further iteration is performed if convergence has not 
TABLE 1: Initial conditions for the IVPs $\left\{Z_{k}^{i}: i=0, \ldots, 3\right\}$.

\begin{tabular}{|c|cccccc|}
\hline$i$ & $G$ & $G^{\prime}$ & $F$ & $F^{\prime}$ & $F^{\prime \prime}$ & $F^{\prime \prime \prime}$ \\
\hline 0 & 0 & $W_{2}(-1)$ & -1 & 0 & $W_{5}(-1)$ & $W_{6}(-1)$ \\
1 & 0 & 1 & 0 & 0 & 0 & 0 \\
2 & 0 & 0 & 0 & 0 & 1 & 0 \\
3 & 0 & 0 & 0 & 0 & 0 & 1 \\
\hline
\end{tabular}

been achieved or greater accuracy is desired. An initial approximate solution is needed to initiate the iterative scheme, and the leading order expressions given in (17) were used for this purpose.

\section{Results and discussion}

We begin with some observations regarding the analytic solutions presented in Section 3. Note that none of the micropolar parameters appear in the leading order solution $f_{0}$ for the stream function given in equation (17); we therefore anticipate that the bulk flow is increasingly insensitive to micropolar effects for increasing mass transfer through the channel walls.

Sample complementary numerical results are given in Figure 1, where computed $U$-velocity profiles for a range of injection Reynolds numbers $(\operatorname{Re}<0)$ are presented, along with the leading order solution $f_{0}^{\prime}$. See in Figure 1(a), the computed profiles for $F^{\prime}$ are close to the leading order analytic solution for the entire range of Re chosen (due to symmetry, profiles are shown over half the channel width only). More detail of the $U$-velocity profiles near the channel centreline $(\eta=0)$ is shown in Figure 1(b). From the figure, observe 
that the computed profiles for $F^{\prime}$ approach the leading order profile for $f_{0}^{\prime}$ as the mass transfer through the channel walls increases, as expected. With regard to the numerical results reported here, we found that the quasilinearisation scheme converged without difficulty, with no more than 5 iterations required in most cases.

Turning to the first order solution for the stream function given in equations (19) and (20), note that the parameters $N_{2}$ and $N_{3}$ do not appear. For the present case, this implies that the bulk flow will quickly become relatively insensitive to changes in these micropolar parameters as the wall mass transfer rate is increased.

In Figure 2, computed $U$-velocity $\left(F^{\prime}\right)$ profiles are presented and compared with the corresponding leading order $\left(f_{0}^{\prime}\right)$ and first order $\left(f_{0}^{\prime}+\epsilon f_{1}^{\prime}\right)$ analytic solutions. Recalling the condition $N_{3}>|\epsilon| N_{1,2}$ (discussed in Section 3), we note that the results presented in Figure 2(a) and 2(b) correspond to $N_{3} /\left(|\epsilon| N_{1,2}\right)=2$ and 5 , respectively. In both cases, the first order analytic solution is in very good agreement with the corresponding computed profile, and the totality of our numerical results for injection indicate that this is always observed provided that the condition $N_{3} /\left(|\epsilon| N_{1,2}\right)>1$ is met.

In principle, the analysis presented in Section 3 is valid for both strong suction $(\operatorname{Re}>0)$ and strong injection $(\operatorname{Re}<0)$. However, note that the corresponding Newtonian flow with large suction is known to have multiple solutions (see Zaturska et al. [16]). Furthermore, our numerical results to date indicate that micropolar flow due to large suction is likely to be more complex than the corresponding Newtonian flow. For example, our preliminary computations for large suction [3] indicate that discontinuities can also occur and are a function of the various micropolar parameters in the model. Currently, our research efforts are directed at analysing this situation in detail and we plan to present our findings in a future paper when completed. 


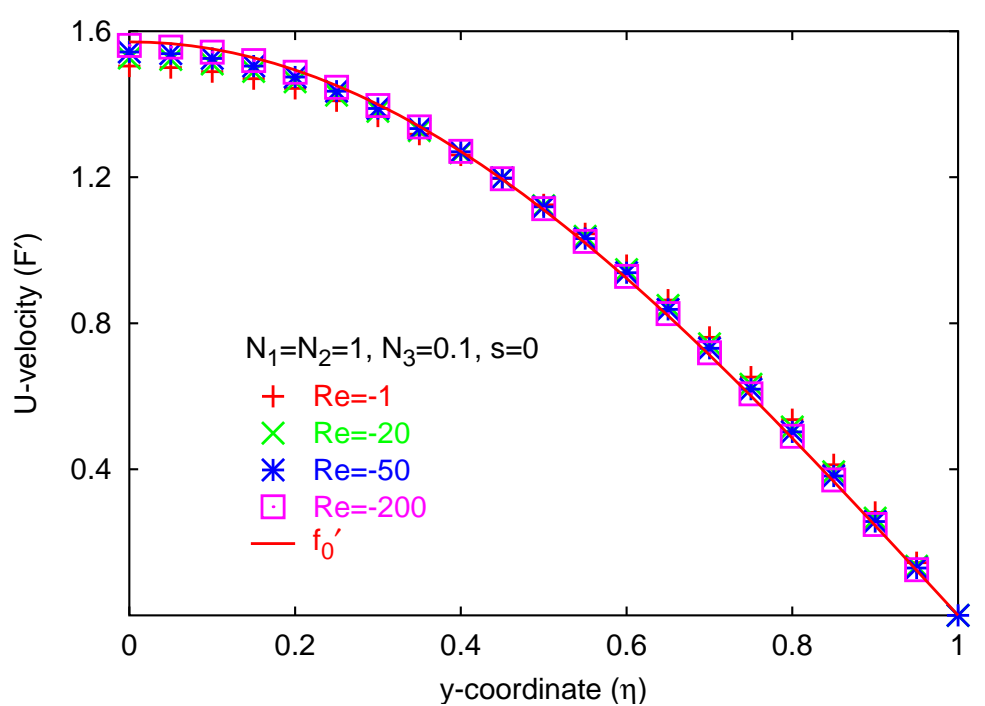

(a)

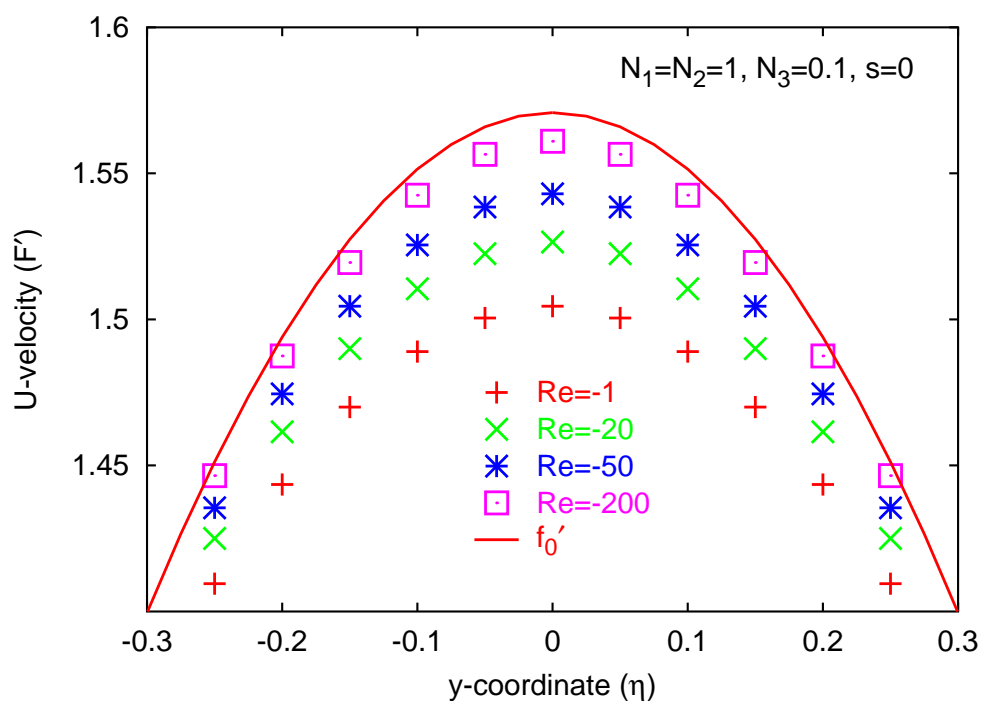

Figure 1: (a) computed $U$-velocity $F^{\prime}$ for $N_{1}=N_{2}=1, N_{3}=0.1$, $s=0$ and $\mathrm{Re}=-1 \rightarrow-200 \mathrm{cf}$. leading order solution $f_{0}^{\prime}$; (b) detail. 


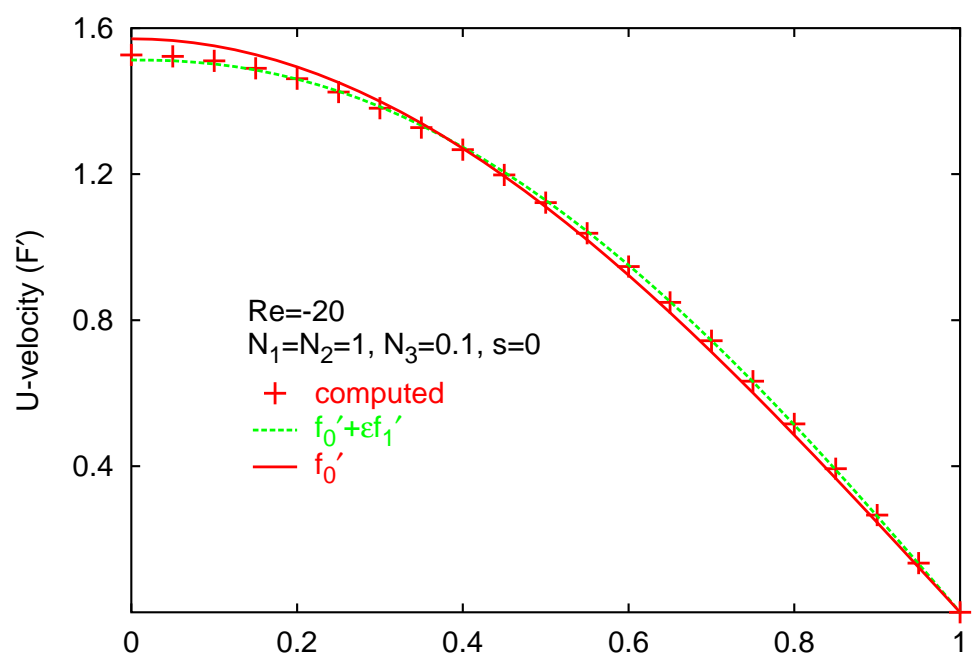

(a)

$y$-coordinate $(\eta)$

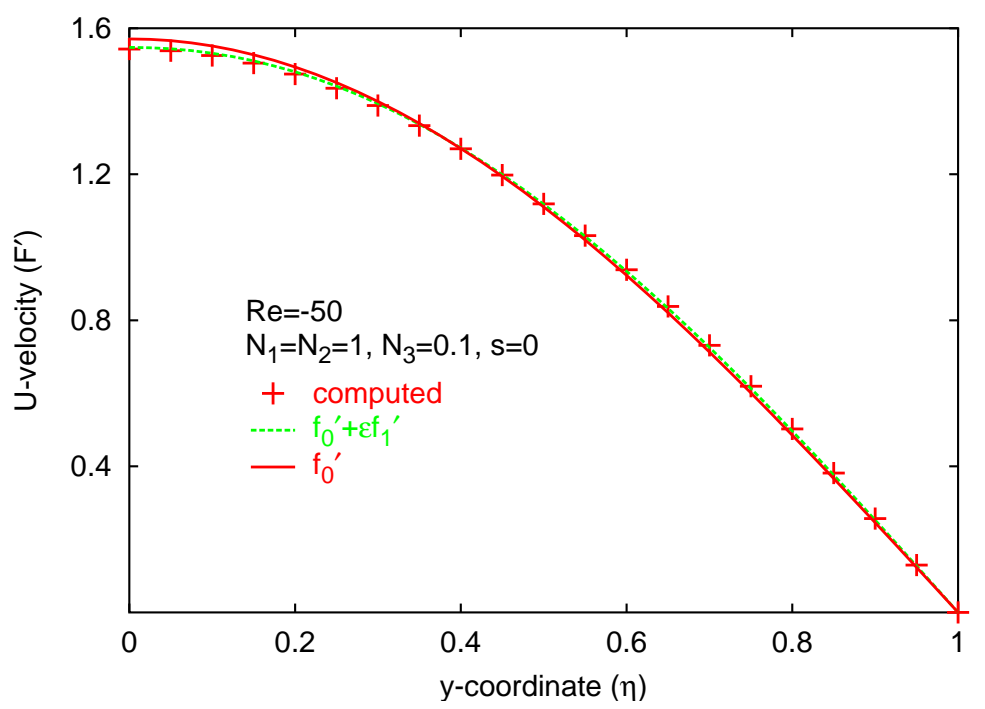

Figure 2: computed $U$-velocity $\left(F^{\prime}\right)$ for $N_{1}=N_{2}=1, N_{3}=0.1$, $s=0$ cf. leading $\left(f_{0}^{\prime}\right)$ and first order $\left(f_{0}^{\prime}+\epsilon f_{1}^{\prime}\right)$ solutions: (a) $\operatorname{Re}=$ -20 ; (b) $\operatorname{Re}=-50$. 


\section{Conclusion}

In this paper we have presented solutions obtained from a perturbation analysis for micropolar flow in a porous channel with large mass transfer through the channel walls, and indicated what these solutions mean in terms of the relative importance of the various micropolar parameters on the bulk flow predictions. Complementary numerical solutions were obtained without difficulty via a quasilinearisation scheme, and very good agreement between the solutions obtained from the perturbation analysis and the computations was observed for strong injection. Our results to date suggest that the case of strong suction is more complex. In future research we intend to analyse this situation in detail, with particular emphasis on testing efficient computational methods for investigating the solution behaviour in 5 parameter $\left(\operatorname{Re}, s, N_{1,2,3}\right)$ space. In addition to numerical algorithm testing, we hope that the results presented here will aid in the understanding of more realistic micropolar fluid applications in related flow setups.

\section{References}

[1] A. S. Berman. Laminar flow in a channel with porous walls. J. Appl. Physics, 24:1232-1235, 1953. C481, C483, C484

[2] A. Desseaux. Influence of a magnetic field over a laminar viscous flow in a semi-porous channel. Int. J. Eng. Sci., 37:1781-1794, 1999. C481

[3] A. Desseaux and N. A. Kelson. Solutions for the flow of a micropolar fluid in a porous channel. In R. L. May, G. F. Fitz-Gerald, and I. H. Grundy, editors, EMAC 2000 
Proceedings: Proc. 4th Biennial Engineering Mathematics and Applications Conference, pages 115-118. The Institution of Engineers, Australia, 2000. C481, C490

[4] A. C. Eringen. Theory of micropolar fluids. J. Mathematics \& Mechanics, 16:1-18, 1966. C481, C485

[5] C. K. Kang and A. C. Eringen. The effect of microstructure on the rheological properties of blood. Bull. Math. Biol., 38:135-158, 1976. C485

[6] N. A. Kelson and A. Desseaux. Flow of a micropolar fluid bounded by a stretching sheet. ANZIAM J., 42(E):C536-560, 2000. C488

[7] N. A. Kelson and A. Desseaux. Effect of surface conditions on flow of a micropolar fluid driven by a stretching sheet. Int. J. Eng. Sci., 39(16):1881-1897, 2001. C481

[8] N. A. Kelson and T. W. Farrell. Micropolar flow over a porous stretching sheet with strong suction or injection. Int. Comm. Heat Mass Transfer, 28(4):479-488, 2001. C481

[9] V. L. Kolpashchikov, N. P. Migun, and P. P. Prokhorenko. Experimental determination of material micropolar fluid constants. Int. J. Eng. Sci., 21(4):405-411, 1983. C485

[10] G. Lukaszewicz. Micropolar fluids: theory and applications. Birkhäuser Boston, 1999. C481, C483

[11] N. P. Migoun and P. P. Prokhorenko. On polar fluid viscosity increase in porous media. In Progress and Trends in Rheology II, pages 244-246, 1988. C485

[12] N. P. Migun. Experimental method of determining parameters characterizing the microstructure of micropolar liquids. J. Eng. Physics, 41(2):220-224, 1981. C485 
[13] P. S. Ramachandran, M. N. Mathur, and S. K. Ojha. Heat transfer in boundary layer flow of a micropolar fluid past a curved surface with suction and injection. Int. J. Engng Sci., 17:625-639, 1979. C483

[14] W. A. Robinson. The existence of multiple solutions for the laminar flow in a uniformly porous channel with suction at both walls. J. Engng. Math., 10:23-40, 1976. C481

[15] S. W. Yuan. Further investigation of laminar flow in channels with porous walls. J. Appl. Physics, 27:267-269, 1956. C481

[16] M. B. Zaturska, P. G. Drazin, and W. H. H. Banks. On the flow of a viscous fluid driven along a channel by suction at porous walls. Fluid Dyn. Res., 4:151-178, 1988. C481, C490

[17] D. Zwillinger. Handbook of differential equations. Academic Press, Boston, 2nd edition, 1992. C487 